\title{
RAPID CHARACTERIZATION OF MAGNETIC SEPARATOR FEED STOCKS IN TITANIUM MINERALS PROCESSING
}

\author{
G. CAVANOUGH ${ }^{\mathrm{a} *}$ and P.N. HOLTHAM ${ }^{\mathrm{b}}$ \\ ${ }^{a}$ CSIRO, QCAT, Puttenvale, Queensland 4069, Australia \\ ${ }^{\mathrm{b}}$ The Julius Kruttschnitt Mineral Research Centre, University of Queensland, \\ Indooroopilly, Queensland 4068, Australia
}

(Received 11 October 2004; Accepted 17 October 2004)

\begin{abstract}
Magnetic separation is widely used in the processing of titanium minerals. The expected mineral recoveries are assessed by performing laboratory magnetic separations of representative samples to determine the distribution of magnetic components. This is an inherently slow process performed on relatively small samples. This paper describes the development of an inductance based device to rapidly determine the mass distribution of the magnetic properties of a titanium mineral sample. The system is best described as an hourglass with mineral flowing from a hopper through a small inductance coil. The impedance of the coil is proportional to the mean magnetic susceptibility of the number of particles within the coil. An algorithm has been developed to determine the percentage of the feed material at each level of magnetic susceptibility from this measurement. The results are identical to those determined using a laboratory magnetic separation, and are obtained in a fraction of the time. Accuracy of the device has been proven by simulation and by testing of plant samples and comparing the results using the new system with measurements made using a magnetic separator.
\end{abstract}

Keywords: Magnetic susceptibility; Magnetic separation; Titanium minerals

\section{INTRODUCTION}

Magnetic characterization of titanium mineral feed stocks is a routine procedure used in the process control and assessment of mineral reserves [1]. Currently, measurements are performed by separating small samples into their constituent magnetic fractions using a laboratory magnetic separator. Results are presented as the percentage of the feed material (by mass) at each level of magnetic separator field strength or speed (both of which are equivalent to magnetic susceptibility).

As an example, the procedure developed by Stradling [2] used an Ore Sorters Permroll separator to process $150-200 \mathrm{~g}$ samples. The Permroll separates material from a feed hopper into magnetic, middling and non-magnetic fractions. Stradling's characterization procedure involves setting the Permroll roll speed to $600 \mathrm{rpm}$,

*Corresponding author. E-mail: garycavanough@burnett.net.au/p.holtham@uq.edu.au 
introducing the sample into the feed hopper and producing a magnetic, middlings and non-magnetic fraction. The middling fraction is recycled until the amount becomes constant. It is then combined with the non-magnetic fraction. The magnetic fraction is removed and weighed and the non-magnetic fraction is returned to the feed hopper. The roll speed is reduced in $50 \mathrm{rpm}$ increments and the process repeated until a final non-magnetic fraction is collected at $150 \mathrm{rpm}$.

As is clear from this description, a significant amount of manual handling is required to perform this task. It takes an operator approximately $40 \mathrm{~min}$ to process one sample. Due to the effort required to perform the initial feed magnetic characterization using the Permroll there has been limited use of Stradling's [2] magnetic separator models to optimise existing plant operations.

The rapid magnetic characterization method described in this paper would result in considerable savings in labour, allow the processing of large samples, enable rapid repeat measurements and would remove the major impediment to adoption of Stradling's magnetic separator models.

\section{MAGNETIC MEASUREMENT SYSTEM}

The magnetic measurement device is best described as an hourglass enclosed in a small coil. Mineral is placed in a hopper and flows through the coil (Fig. 1). Both time and coil inductance are recorded as the sample flows through the device. Measurements are made 10 times per second and results are processed by a computer. The details of the measurement method are covered in full in Cavanough and Holtham [3].

The main components are:

1. hopper to deliver mineral;

2. a small send/receive inductance coil;

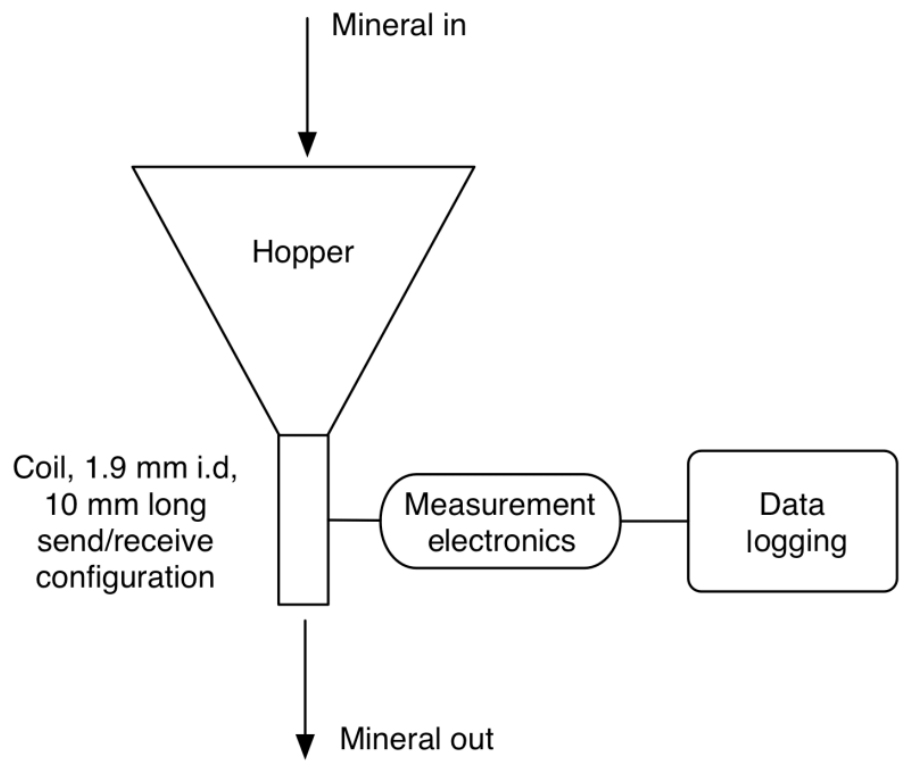

FIGURE 1 Schematic of measurement system. 
3. the electronic measurement systems to convert the changes in coil inductance to units of magnetic susceptibility;

4. a computer and data logging system to allow sample acquisition at a frequency $10 \mathrm{~Hz}$;

5. software to calculate the mean and standard deviation of the magnetic susceptibility for the recorded data and then determine the mass distribution of the magnetic susceptibility.

The calculation of the mean and standard deviation is the most significant component of the system and will therefore be the focus of this paper.

\section{CALCULATION OF THE MEAN AND STANDARD DEVIATION OF THE MAGNETIC SUSCEPTIBILITY}

The magnetic susceptibility of mineral particles inside the coil is given by:

$$
\Sigma_{\chi}=\Sigma_{\chi_{i}} v_{i}
$$

where $\Sigma_{\chi}$ is the measured magnetic susceptibility and $v_{i}$ is the volume fraction of mineral with a susceptibility of $\chi_{I}[4-6]$.

Stated more simply, the measurement will be the average magnetic susceptibility of the particles contained within the coil. For example if the coil held three particles with magnetic susceptibilities of $a_{1}, a_{2}$ and $a_{3}$, the measured magnetic susceptibility would be: $\left(a_{1}+a_{2}+a_{3}\right) / 3$. Continuing with this reasoning will enable definition of the types of sampling that will occur. If the whole sample consisted of nine particles represented by group $\mathrm{A}$ such that $\mathrm{A}=\left\{a_{1}, a_{2}, a_{3}, a_{4}, a_{5}, a_{6}, a_{7}, a_{8}, a_{9}\right\}$ with each element of $A$ being equal to the magnetic susceptibility of a unique particle, then the particles can be normally sampled, over sampled or under sampled as described below.

\subsection{Normal Sampling}

Three particles are contained within the coil and a measurement is taken every $0.01 \mathrm{~s}$. The particles of A are flowing though the coil at a rate of three particles every $0.01 \mathrm{~s}$. Therefore three measurements are taken as the mineral flows through the coil. Each measurement can be represented as an element of the set $A_{g}$. The elements of $A_{g}$ are dependent on the order in which minerals flow through the coil. For example, if the particles flow through the coil in the order of one to nine then:

$$
A_{g}=\left\{\frac{\left(a_{1}+a_{2}+a_{3}\right)}{3}, \frac{\left(a_{4}+a_{5}+a_{6}\right)}{3}, \frac{\left(a_{7}+a_{8}+a_{9}\right)}{3}\right\}
$$

\subsection{Over Sampling}

Three particles are contained within the coil and a measurement is taken every $0.01 \mathrm{~s}$. The particles of A are flowing though the coil at a rate of two particles every $0.01 \mathrm{~s}$. 
Therefore four measurements are taken as the mineral flows through the coil (neglecting the sample where the coil is not full). Each measurement can be represented as an element of the set $A_{\mathrm{go}}$. The elements of $A_{\mathrm{go}}$ are dependent on the order in which minerals flow through the coil. For example, if the particles flow through in the order of one to nine then:

$$
A_{\mathrm{go}}=\left\{\frac{\left(a_{1}+a_{2}+a_{3}\right)}{3}, \frac{\left(a_{3}+a_{4}+a_{5}\right)}{3}, \frac{\left(a_{5}+a_{6}+a_{7}\right)}{3}, \frac{\left(a_{7}+a_{8}+a_{8}\right)}{3}\right\}
$$

\subsection{Under Sampling}

Three particles are contained within the coil and a measurement is taken every $0.01 \mathrm{~s}$. The particles of A are flowing though the coil at a rate of six particles every $0.01 \mathrm{~s}$. Therefore some of the particles are not measured and only two measurements are taken as the mineral flows through the coil. Each measurement can be represented as an element of the set $A_{\mathrm{gu}}$. The elements of $A_{\mathrm{gu}}$ are dependent on the order in which minerals flow through the coil. For example, if the particles flow through in the order of one to nine then:

$$
A_{\mathrm{gu}}=\left\{\frac{\left(a_{1}+a_{2}+a_{3}\right)}{3}, \frac{\left(a_{7}+a_{8}+a_{9}\right)}{3}\right\}
$$

\section{DETERMINATION OF MEAN AND STANDARD DEVIATION FOR A SAMPLE}

In the following sections the method to determine the mean and standard deviation of the susceptibility of a real sample will be discussed for the cases of normal, under and over sampling.

\subsection{Normal Sampling}

The mineral sample consists on $n$ particles of differing magnetic susceptibility as represented by set $A$ :

$$
A=\left\{a_{1}, a_{2}, a_{3}, \ldots, a_{n}\right\}
$$

Let $s$ denote the number of particles that are within the field of the coil when a measurement is made. Therefore the signal from the coils can be represented by $A_{g}$ :

$$
A_{g}=\left\{\frac{\sum a_{1 \rightarrow s}}{s}, \frac{\sum a_{s+1 \rightarrow 2 s}}{s}, \frac{\sum a_{2 s+1 \rightarrow 3 s}}{s}, \ldots, \frac{\sum a_{\left(n s^{-1}-1\right) \times s \rightarrow n s^{-1} \times s}}{s}\right\}
$$


Since both $A$ and $A_{g}$ contains the same particles the means are the same, hence:

$$
\bar{A}=\bar{A}_{g}
$$

The sum of squares and the sum of $A$ are:

$$
\begin{aligned}
& \sum A^{2}=a_{1}^{2}+a_{2}^{2}+a_{3}^{2} \ldots a_{n}^{2} \\
& \sum A=a_{1}+a_{2}+a_{3} \ldots a_{n}=n \times \bar{A}_{g}
\end{aligned}
$$

The sum of squares of $A_{g}$ is:

$$
\begin{aligned}
& \sum A_{g}^{2}=\frac{\sum a_{1 \rightarrow s}^{2}}{s^{2}}+\frac{\sum a_{s+1 \rightarrow 2 s}^{2}}{s^{2}}+\frac{\sum a_{2 s+1 \rightarrow 3 s}^{2}}{s^{2}} \ldots \frac{\sum a_{\left(n s^{-1}-1\right) \times s \rightarrow n s^{-1} \times s}^{2}}{s^{2}} \\
\Rightarrow & \sum A_{g}^{2}=\frac{a_{1}^{2}+a_{2}^{2}+a_{3}^{2} \ldots a_{n}^{2}}{s^{2}}+\frac{\bar{a}_{x} a_{y} \times{ }_{s} \mathrm{P}_{2} \times(n / s)}{s^{2}} \\
\Rightarrow & \sum A_{g}^{2}=\frac{\sum A^{2}}{s^{2}}+\frac{\bar{a}_{x} a_{y} \times{ }_{s} \mathrm{P}_{2} \times(n / s)}{s^{2}}
\end{aligned}
$$

Rearranging gives the sums of squares of $A$ :

$$
\Rightarrow \sum A^{2}=s^{2} \sum A_{g}^{2}-\frac{\overline{a_{x} a_{y}} \times{ }_{s} \mathrm{P}_{2} \times n}{s}
$$

where: $\overline{a_{x} a_{y}}=$ the mean value of multiplying all combinations of 2 particles together; ${ }_{s} \mathrm{P}_{2}=$ the number of permutations of 2 particles from $s$ particles.

The sum of multiplying each particle of $A_{g}$ with the next particle of $A_{g}$ is represented by:

$$
\begin{array}{r}
\sum A_{a \times b}=\frac{\left(a_{1 \rightarrow s}\right)^{2}}{s^{2}} \times \frac{\left(a_{s+1 \rightarrow 2 s}\right)^{2}}{s^{2}}+\frac{\left(a_{s+1 \rightarrow 2 s}\right)^{2}}{s^{2}} \times \frac{\left(a_{2 s+1 \rightarrow 3 s}\right)^{2}}{s^{2}} \cdots \\
+\frac{\left(a_{\left(n s^{-1}-2\right) \times s \rightarrow\left(n s^{-1}-1\right) \times s}\right)^{2}}{s^{2}} \times \frac{\left(a_{\left(n s^{-1}-1\right) \times s \rightarrow\left(n s^{-1} \times s\right.}\right)^{2}}{s^{2}}
\end{array}
$$

From this equation the value of $\overline{a_{x} a_{y}}$ is:

$$
\overline{a_{x} a_{y}}=\frac{s \times \sum A_{a \times b}}{(n-s)}
$$

Substitution gives:

$$
\sum A^{2}=s^{2} \sum A_{g}^{2}-\frac{\sum A_{a \times b} \times{ }_{s} P_{2} \times n}{n-s}
$$


and the standard deviation is:

$$
\begin{aligned}
S D & =\sqrt{\frac{n \times \sum A^{2}-n^{2} \times\left(\overline{\sum A_{g}}\right)^{2}}{n(n-1)}} \\
\Rightarrow S D & =\sqrt{\frac{\sum A^{2}-n \times\left(\overline{\sum A_{g}}\right)^{2}}{(n-1)}}
\end{aligned}
$$

\subsection{Over Sampling}

The mineral sample consists of $n$ particles of differing magnetic susceptibility as represented by set $A$ :

$$
A=\left\{a_{1}, a_{2}, a_{3}, \ldots, a_{n}\right\}
$$

Let $s$ denote the number of particles that are within the field of the coil when a measurement is taken. However the sampling time is faster than the time required for the coil volume to be replaced and some particles are measured twice. Therefore, the signal from the coils can be represented by $A_{\mathrm{go}}$ :

$$
A_{g}=\left\{\frac{\sum a_{1 \rightarrow s}}{s}, \frac{\sum a_{s-c+1 \rightarrow 2 s-c}}{s}, \frac{\sum a_{2 s-c+1 \rightarrow 3 s-c}}{s}, \ldots, \frac{\sum a_{\left(\left(n s^{-1}-1\right) \times s\right)-c+1 \rightarrow\left(n s^{-1} \times s\right)-c}}{s}\right\}
$$

Assume $c<s$ then the means are approximately equal:

$$
\bar{A} \approx \overline{A_{\mathrm{go}}}
$$

The sum of squares and the sum of $A$ are:

$$
\begin{aligned}
& \sum A^{2}=a_{1}^{2}+a_{2}^{2}+a_{3}^{2} \ldots a_{n}^{2} \\
& \sum A=a_{1}+a_{2}+a_{3} \ldots a_{n}=n \times \overline{A_{\mathrm{go}}}
\end{aligned}
$$

The sum of squares of $A_{\mathrm{go}}$ is:

$$
\begin{aligned}
& \sum A_{\mathrm{go}}^{2}=\frac{\sum a_{1 \rightarrow s}^{2}}{s^{2}}+\frac{\sum a_{s-c+1 \rightarrow 2 s-c}^{2}}{s^{2}}+\frac{\sum a_{2 s-c+1 \rightarrow 3 s-c}^{2}}{s^{2}} \ldots \frac{\sum a_{\left(\left(n s^{-1}-1\right) \times s\right)-c+1 \rightarrow\left(n s^{-1} \times s\right)-c}^{2}}{s^{2}} \\
\Rightarrow & \sum A_{\mathrm{go}}^{2}=\frac{a_{1}^{2}+a_{2}^{2}+a_{3}^{2} \ldots a_{n}^{2}}{s^{2}}+\frac{a_{c 1}^{2}+a_{c 2}^{2}+c_{c 3}^{2} \ldots a_{c \ldots}^{2}}{s^{2}}+\frac{\overline{a_{x}} a_{y} \times{ }_{s} \mathrm{P}_{2} \times(n / \dot{s})}{s^{2}} \\
\Rightarrow & \sum A_{\mathrm{go}}^{2}=\frac{\sum A^{2}}{s^{2}}+\frac{\sum C^{2}}{s^{2}}+\frac{\overline{a_{x} a_{y}} \times{ }_{s} \mathrm{P}_{2} \times n / s}{s^{2}}
\end{aligned}
$$


Rearranging gives the sums of squares of $A$ :

$$
\Rightarrow \sum A^{2}=s^{2} \sum A_{\mathrm{go}}^{2}-\sum C^{2}-\frac{\overline{a_{x} a_{y}} \times{ }_{s} \mathrm{P}_{2} \times n}{s}
$$

where: $\overline{a_{x} a_{y}}=$ the mean value of multiplying all combinations of 2 particles together. ${ }_{s} \mathrm{P}_{2}=$ the number of permutations of 2 particles from $\mathrm{s}$ particles. $\sum C^{2}=$ the sum of all the common particle values.

The sum of multiplying each particle of $A_{\text {go }}$ with the next particle of $A_{\text {go }}$ is represented by:

$$
\begin{gathered}
\sum A_{a \times b}=\frac{\left(a_{1 \rightarrow s}\right)^{2}}{s^{2}} \times \frac{\left(a_{s-c+1 \rightarrow 2 s-c}\right)^{2}}{s^{2}}+\frac{\left(a_{2 s-c+1 \rightarrow 3 s-c}\right)^{2}}{s^{2}} \times \frac{\left(a_{3 s-c+1 \rightarrow 4 s-c}\right)^{2}}{s^{2}} \cdots \\
+\frac{\left(a_{\left(n s^{-1}-2\right) \times s-c+1 \rightarrow\left(n s^{-1}-1\right) \times s-c}\right)^{2}}{s^{2}} \times \frac{\left(a_{\left(n s^{-1}-1\right) \times s-c+1 \rightarrow n s^{-1} \times s-c}\right)^{2}}{s^{2}} \\
\sum A_{a \times b}=\frac{\sum C^{2}}{s^{2}}+\frac{\left(s^{2}-C_{n}\right) \times \overline{a_{x} a_{y}} \times(n-s / s)}{s^{2}}
\end{gathered}
$$

where $C_{n}=$ the number of common particles in any group.

If $C_{n} \ll S^{2}$ the value of $\overline{a_{x} a_{y}}$ is:

$$
\overline{a_{x} a_{y}}=\frac{s}{n-s} \times\left\{\sum A_{a \times b}-\frac{\sum C^{2}}{s^{2}}\right\}
$$

Substitution gives:

$$
\begin{aligned}
\sum A^{2}= & s^{2} \times \sum A_{\mathrm{go}}^{2}-\sum C^{2}-\overline{a_{x} a_{y}} \times{ }_{s} \mathrm{P}_{2} \times \frac{n}{s} \\
\sum A^{2}= & s^{2} \times \sum A_{\mathrm{go}}^{2}-\sum C^{2}-\frac{s}{n-s} \times\left\{\sum A_{a \times b}-\frac{\sum C^{2}}{s^{2}}\right\} \times{ }_{s} \mathrm{P}_{2} \times \frac{n}{s} \\
\sum A^{2}= & s^{2} \times \sum A_{\mathrm{go}}^{2}+\sum C^{2}\left\{\frac{n_{s} \mathrm{P}_{2}}{(n-s) s^{2}}-1\right\}-\frac{\sum A_{a \times b} \times{ }_{s} \mathrm{P}_{2} \times n}{n-s} \\
\sum A^{2}= & s^{2} \times \sum A_{\mathrm{go}}^{2}+\sum C^{2} \times\left(\frac{n \times s \times(s-1)}{(n-s) \times s^{2}}-1\right)-\sum A_{a \times b} \times{ }_{s} \mathrm{P}_{2} \times 2 \\
& \left.\left.\times\left\{s^{2} \times \sum A_{\mathrm{go}}^{2}\right\}\right\rangle\right\rangle\left\{\sum C^{2} \times\left(\frac{n \times s \times(s-1)}{(n-s) \times s^{2}}-1\right)\right\}
\end{aligned}
$$

Therefore:

$$
\sum A^{2}=s^{2} \times \sum A_{\mathrm{go}}^{2}-\frac{\sum A_{a \times b} \times{ }_{s} \mathrm{P}_{2} \times n}{n-s}
$$


and the standard deviation is:

$$
\Rightarrow S D=\sqrt{\frac{\sum A^{2}-n \times\left(\overline{\sum A_{g}}\right)^{2}}{(n-1)}}
$$

\subsection{Under Sampling}

The mineral sample consists of $n$ particles of differing magnetic susceptibility as represented by set $A$ :

$$
A=\left\{a_{1}, a_{2}, a_{3}, \ldots, a_{n}\right\}
$$

Let $s$ denote the number of particles that are within the field of the coil when a measurement is taken. However the sampling time is slower than the time required for the coil volume to be replaced and not all particles are measured. Therefore, the signal from the coils can be represented by $A_{\mathrm{gu}}$ :

$$
A_{\mathrm{gu}}=\left\{\frac{\sum a_{1 \rightarrow s}}{s}, \frac{\sum a_{2 s+1 \rightarrow 3 s}}{s}, \ldots, \frac{\sum a_{\left(n s^{-1}-1\right) \times s \rightarrow n s^{-1} \times s}}{s}\right\}
$$

If a significant number of particles are contained in $A_{\mathrm{gu}}$ it will be a representation of the set A. Therefore:

$$
\sum A^{2}=s^{2} \times \sum A_{\mathrm{go}}^{2}-\frac{\sum A_{a \times b} \times{ }_{s} \mathrm{P}_{2} \times n}{n-s}
$$

and the standard deviation is:

$$
\Rightarrow S D=\sqrt{\frac{\sum A^{2}-n \times\left(\overline{\sum A_{g}}\right)^{2}}{(n-1)}}
$$

Note that for under sampling $n$ is the number of samples actually measured, not the total number of particles.

\section{VERIFICATION OF MEAN AND STANDARD DEVIATION BY SIMULATION}

Mathematical simulation was used to test the accuracy of the standard deviation calculations. Verification of the mean value was not required as the proof above indicates that the mean value will always be correct. The procedure was:

- Generate 10 sets of 50000 random numbers with a normal distribution (each representing a particle).

- Calculate the standard deviations of the data. 
- Group the data into 100 element sets.

- Calculate the standard deviations using the basic equation with $s=100$ and $n=50000$.

- Group the data in 100 element sets with a 20 element overlap between sets with $s=100$ and $n=50000$.

- Calculate the standard deviations using the over sampling equation.

- Group the data in 100 element sets and discard every second set.

- Calculate the standard deviations using the under sampling equation with $s=100$ and $n=25000$.

All simulations resulted in a calculated standard deviation within \pm 1 of the true value.

\section{GENERATION OF DISTRIBUTION OF MAGNETIC COMPONENTS}

Magnetic characterization data of a mineral feed stock is expressed as a percentage of the feed material passing a given magnetic susceptibility (Fig. 2). Once the mean and standard deviation of the distribution are known generation of curves as shown in Fig. 2 is a straight forward operation. The procedure is to generate a normal probability distribution with the calculated mean and standard deviation. From this curve the cumulative percentages for any susceptibility values can be calculated.

\subsection{Testing Plant Samples}

Three samples from Stradling's [2] test work were used to verify the characterization method. These samples were from a plant survey and consisted of feed stocks to a primary and secondary magnetic separator circuits. Stradling reported the

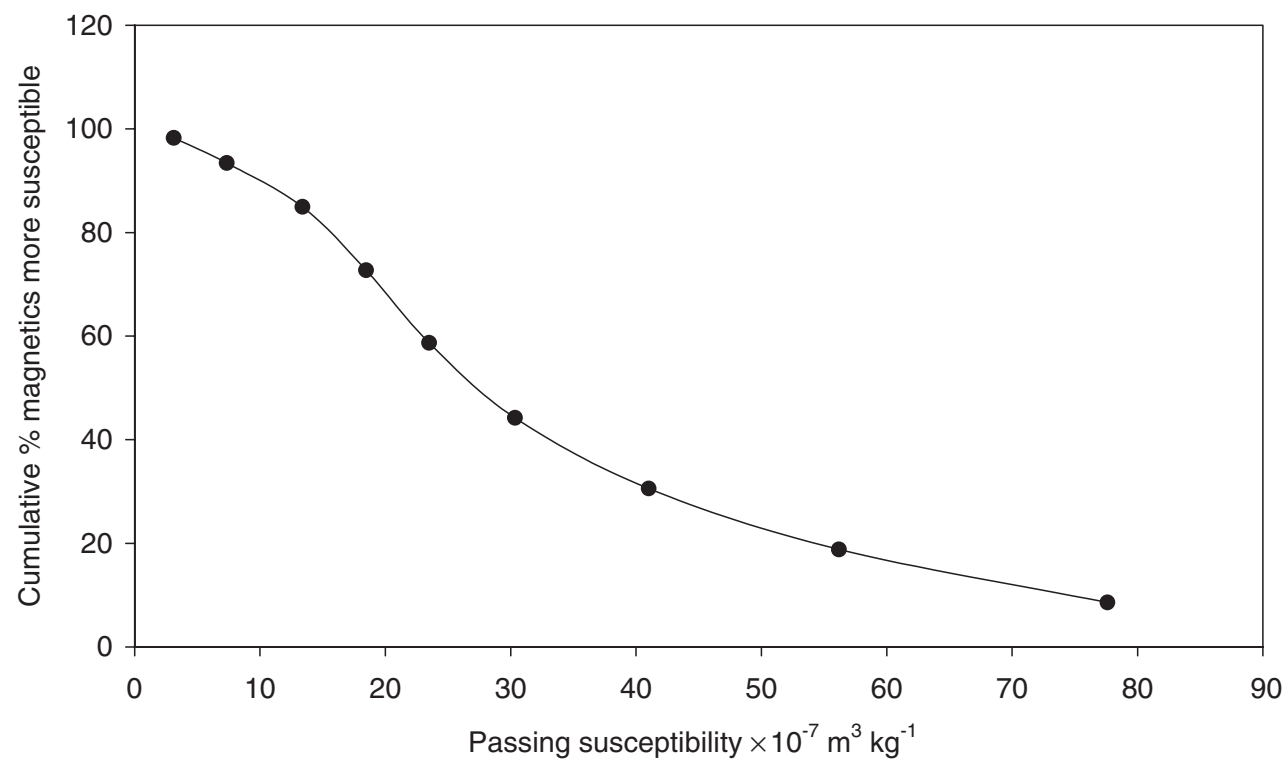

FIGURE 2 Typical Permroll separation results. 
TABLE I Data used to derive group size and total number of members

\begin{tabular}{lc}
\hline Sample mass $(\mathrm{g})$ & 12.28 \\
Mass flow rate $\left(\mathrm{g} \mathrm{s}^{-1}\right)$ & 0.04 \\
Mean grain diameter $(\mathrm{mm})$ & 0.18 \\
Mean grain cross-sectional area $\left(\mathrm{mm}^{2}\right)$ & $2.8 \times 10^{-2}$ \\
Mean grain mass $(\mathrm{g})$ & $8.5 \times 10^{-6}$ \\
Coil length $(\mathrm{mm})$ & 10 \\
Coil cross-sectional area $\left(\mathrm{mm}^{2}\right)$ & 2.8 \\
\hline
\end{tabular}

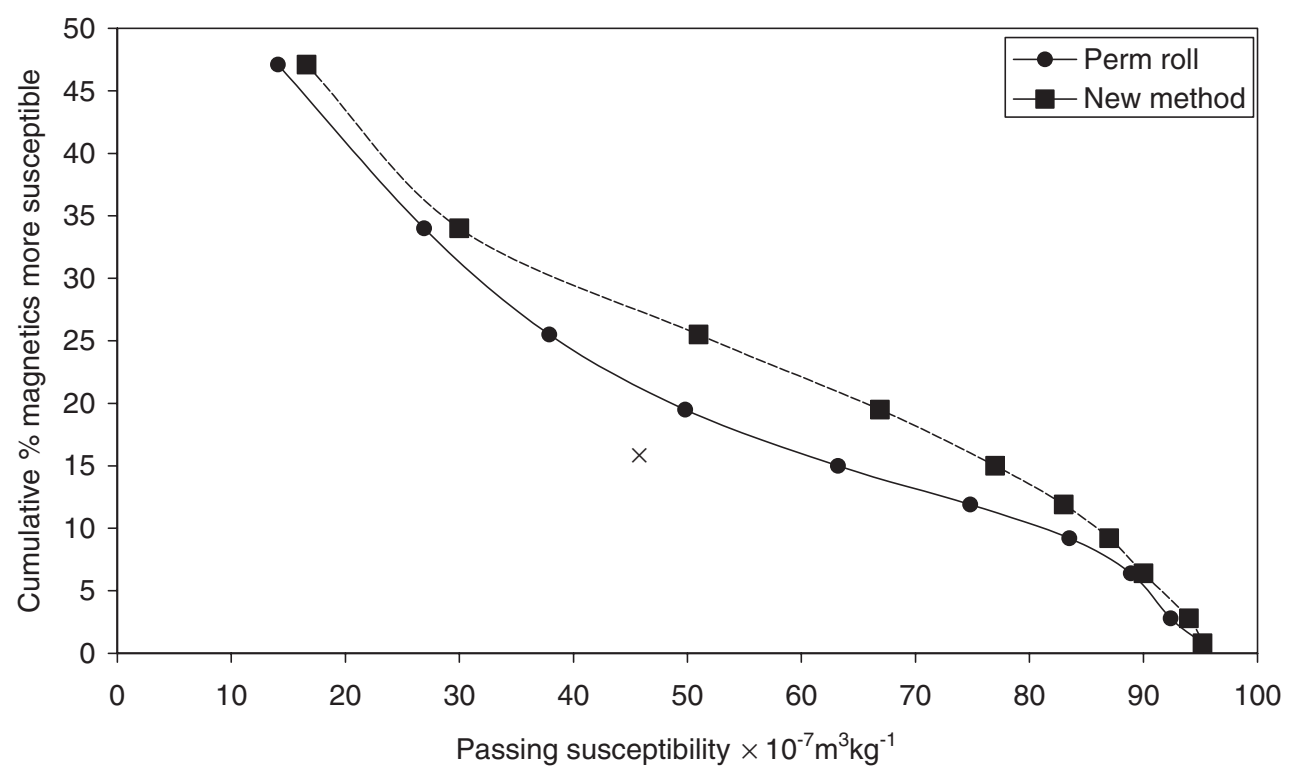

FIGURE 3 Comparison of results for sample PX16/13 feed.

cumulative percent passing curves from the Permroll fractionation of these samples. This allowed comparison with curves calculated using the method described here.

The calculation requires the constants of group size and total number of members. These values were calculated based on the data shown in Table I. From the coil and grain cross-sectional areas it can be seen that the coil will hold layers of approximately 100 grains. With a mineral flow rate of $0.04 \mathrm{~g} \mathrm{~s}^{-1}$ the discharge rate is approximately 4700 grains s$^{-1}$, hence the coil discharges 47 layers of grains per second.

The coil is $10 \mathrm{~mm}$ long, therefore it holds 67 layers of grains, hence the group size can be taken as 67 . With 47 layers discharging per second, there will be 20 layers common to each group. The total number of members, $n$ is given by $n=s+(t \times(s-c))$, where $n$ is the total number of members, $s$ is the group size, $t$ the number of samples taken while mineral is present in the coil $(10 \mathrm{~Hz}$ sampling rate) and $c$ the number of common elements to intersecting groups.

Samples were measured and the distribution of magnetic components calculated using the constants derived above. Calculated results compared favourably with those reported by Stradling (Figs. 3-5). 


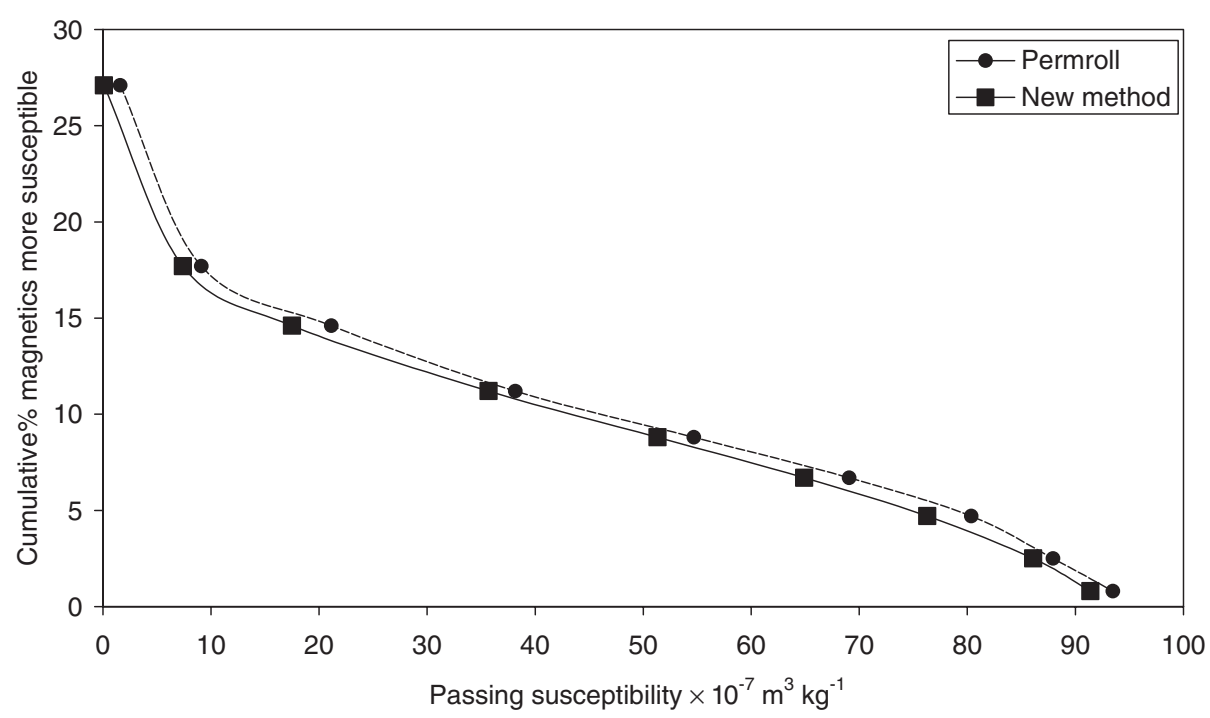

FIGURE 4 Comparison of results for sample SX11/16 feed.

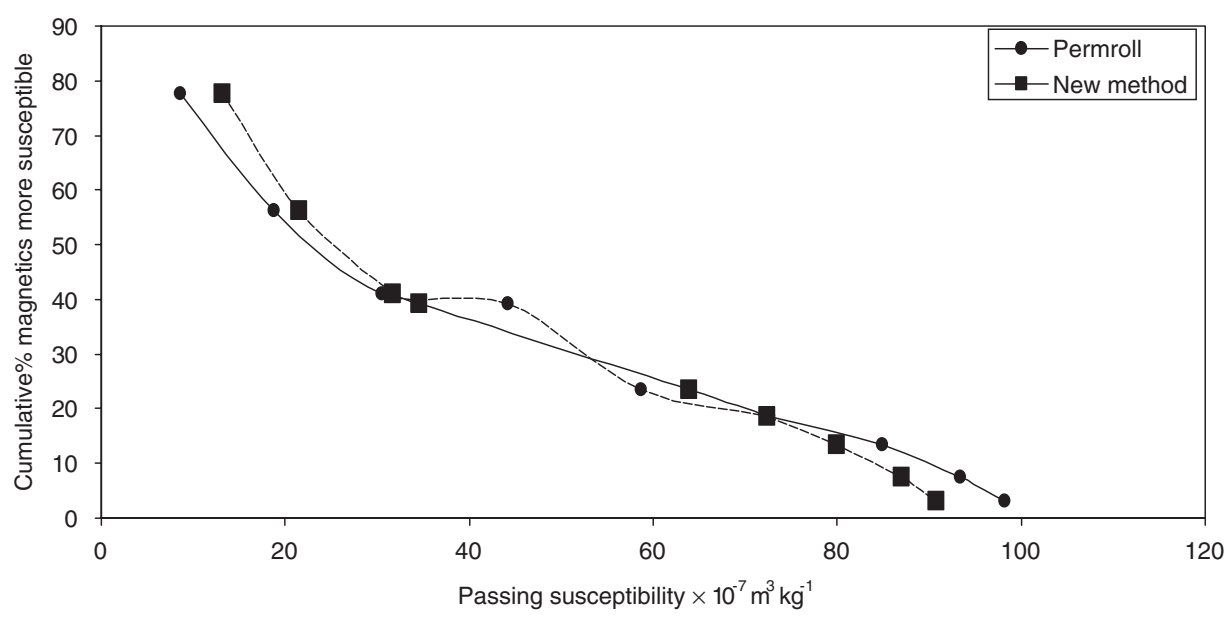

FIGURE 5 Comparison of results for sample JAP18* feed.

\section{CONCLUSION}

A system has been developed to determine the distribution of magnetic components in a titanium minerals feed stock sample. This system has been proven through mathematical derivation of the operating principal, simulation and testing of plant samples.

This system provides the same results as obtained by using a Permroll separation with the following advantages over Permroll separation:

- No sample mass measurements are required.

- The measurement takes approximately $1 \mathrm{~min}$ per sample compared to $40 \mathrm{~min}$ per sample for a Permroll separation. 


\section{Acknowledgements}

The financial support of Cable Sands, Iluka Resources and Tiwest through AMIRA Project P255 is gratefully acknowledged.

\section{References}

[1] S.P. Panov, J.B. Metson and N. Batchelor, Benefication of New Zealand ilmeno-magnetite. The AusIMM Proceedings, 1 (2000).

[2] A. Stradling, Mathematical models of dry high-intensity magnetic separators, PhD thesis, University of Queensland, Brisbane (1991).

[3] G. Cavanough and P.N. Holtham, On-line measurement on magnetic susceptibility in titanium minerals processing. The AusIMM Proceedings, 30(2) (2001), 1-6.

[4] D.M. Hopstock, Mineral Processing Handbook (N.L. Weiss, ed.), AIME, New York (1985), pp. 6-26.

[5] T. Nagata, Rock Magnetism, Maruzen, Tokyo (1961).

[6] F. Janak, Determination of anistropy of magnetic susceptibility of rocks. Stud. Geoph. Geod., 9 (1965), 290-301. 